\title{
IngESTION OF MALE HEMOLYMPH BY FEMALE SAGEBRUSH CRICKETS: No EVIDENCE OF MALE-DERIVED ANTI-APHROdISIACS
}

\author{
CARIE B. WEDdLE $\uparrow$ JAMIE S. AlLEN $\downarrow$ Hilton M. JonES \\ JOHN K. SAKALUK $\downarrow$ SCOTT K. SAKALUK \\ DEPARTMENT OF BIOLOGICAL SCIENCES \ ILLINOIS STATE UNIVERSITY \\ NORMAL $\uparrow \mathrm{IL}$
}

\begin{abstract}
$\downarrow$ ABSTRACT
Male sagebrush crickets, Cyphoderris strepitans, offer an unusual nuptial food gift to females during copulation: females are permitted to feed on the hind wings of males and ingest hemolymph that flows from the resulting wounds. Johnson et al. (1999) showed that females experimentally precluded from wing feeding during initial copulations were more receptive to subsequent matings than females allowed to obtain a blood meal during initial copulations. The present study was designed to test the hypothesis that substances contained in the hemolymph of males and ingested by females during copulation function to decrease female receptivity to further matings, and thereby constitute male manipulation. To test this hypothesis, females were allowed to mate with an initial virgin male whose hind wings had been surgically removed to prevent wing feeding. Following these initial copulations, females were fed one of the following substances: male hemolymph, female hemolymph, or cricket Ringer's solution. Immediately following ingestion of the prescribed substances, females were given the opportunity to remate with novel virgin males whose hind wings had been left intact, and their latency to remating used as a measure of their sexual receptivity. There was no significant difference in latency to remating across treatments, suggesting no effect of hemolymph ingestion on female receptivity. The disparity between the results of the previous study (Johnson et al. 1999) and those
\end{abstract}

of the present one suggest that the nutritional contribution derived through wing feeding, rather than male manipulation of female receptivity via substances in the hemolymph, affect remating propensity of female sagebrush crickets.

\section{$\downarrow$ INTRODUCTION}

In various insect species, females mate with multiple males and are able to exert greater control over the process of reproduction than males, because females maintain direct control over the transfer of sperm and the use of stored sperm for subsequent fertilizations (Thornhill and Alcock 1983; Sakaluk 1984; Sakaluk and Eggert 1996; Eberhard 1996). From an evolutionary perspective, such cryptic female choice is in direct conflict with the reproductive interests of males. While females may benefit by being selective of the potential sires of their offspring, male reproductive success is contingent upon maximizing the returns from their gametic investment by ensuring that their sperm are used for fertilizations.

This direct evolutionary conflict between the sexes over the use of sperm for fertilizations has led to the evolution of male counter-strategies to cryptic female mating preferences in many species. Such male counter strategies include: mate guarding, mate sequestering, multiple or forced copulations, or the use of specific anti-receptivity devices (such as 
mating plugs) or anti-receptivity substances (Sakaluk 1991; Andersson and Iwasa 1996; Sakaluk et al. 1995). The use of anti-aphrodisiacs, such as hormones transferred from the male to the female during copulation, has been reported for several insect species (Eberhard 1996).

Male sagebrush crickets, Cyphoderris strepitans, offer an unusual nuptial food gift to females during copulation. Females are allowed to feed on the fleshy hind wings of males and to ingest hemolymph that oozes from the resultant, non-fatal wounds. Because only a portion of the hind wing material is consumed in a given copulation, males are not precluded from mating again (Dodson et. al 1983). In a recent study by Johnson et al. (1999), female $C$. strepitans were given the opportunity to mate with virgin males in an initial mating trial in which males were subjected to one of two experimental treatments: 1) males' hind wings were left intact such that copulatory wing feeding was possible; 2) males' hind wings were surgically removed to prevent copulatory wing feeding. Following this initial copulation, females were allowed the opportunity to remate with a virgin male with intact hind wings, and latency to remating was recorded as a measure of female receptivity to subsequent copulations. Females that were experimentally prevented from wing feeding during initial copulations were significantly more receptive to subsequent copulations than females who were allowed to wing feed during their initial mating.

Johnson et al. (1999) interpreted these results as evidence for cryptic female choice, in which females receiving inadequate nourishment from males at mating seek out additional matings with males who offer a greater nutritional investment. Alternatively, the fact that females who were allowed to wing feed during initial copulations were less receptive to matings with subsequent males could be interpreted as evidence of male manipulation (Johnson et al. 1999). This study was designed to test the hypothesis that chemical substances contained in the hemolymph of males and ingested by females during copulation, function to decrease female receptivity to subsequent copulations and thereby protect the paternity of investing males.

To test this hypothesis, females captured early in the breeding season (May-June 2000) were mated with an initial virgin male whose hind wings had been surgically removed to prevent wing feeding. Following successful initial copulation, females were fed either hemolymph obtained from adult males, hemolymph obtained from adult females, or cricket
Ringer's solution. Immediately following the prescribed feeding treatment, females were given the opportunity to remate with a novel virgin male with intact hind wings. The "male manipulation" hypothesis predicts that ingestion of adult male hemolymph should increase female latency to subsequent copulation relative to that of females who ingest either the hemolymph of adult females or cricket Ringer's solution.

\section{MethodS}

The study was conducted early in the breeding season (mid May) at the University of Wyoming-National Park Service Research Center, where previous studies of this species have been conducted (Snedden and Sakaluk 1992; Eggert and Sakaluk 1994; Sakaluk et al. 1995). Adult males and adult females were collected from several populations within Grand Teton National Park and transported to the research center. Experimental subjects were maintained at the research center according to standard procedures (Snedden and Sakaluk 1992; Eggert and Sakaluk 1994). All males used in the experiment were virgins, as indicated by intact hind wings. The mating status of females was unknown.

Twenty-four hours after initial capture, females were randomly assigned to initial mating trials with a virgin male whose hind wings had been surgically removed to prevent wing feeding. Surgically altered males were allowed 24 hours recovery prior to use in mating trials. Initial mating trials were established in small, glass viewing jars equipped with a short stick as a calling perch for males. Pairs were established early in the evening when the crickets normally become sexually active and mating behaviors were monitored over the next six hours via direct observation. Females that did not mate successfully during the first night were allowed the opportunity to mate the next night and every night thereafter until successful initial copulation had occurred or the study had ended. No food or water was provided during mating trials.

Immediately following successful copulation with the initial male, experimental treatments were established in which females were allowed to consume $20 \mu \mathrm{l}$ of one of the following substances: 1) hemolymph obtained from an adult male; 2) hemolymph obtained from an adult female; 3) cricket Ringer's solution. All treatments were pipetted onto a small piece of puffed rice, which readily absorbed the liquid, and presented to females 
on the tip of a dissecting probe. Females readily consumed food items presented in this manner.

Immediately following the ingestion of their prescribed substance, females were assigned to subsequent mating trials with novel virgin males whose hind wings were left intact. Experimental pairs were placed into specially constructed Plexiglas viewing chambers divided into two equal compartments $(10 \times 6.8 \times 4.4 \mathrm{~cm})$ that allowed timelapse video monitoring of subsequent mating trials. Each compartment was equipped with a calling perch in the form of a short stick. Females that had not mated successfully by dawn were paired with another virgin male for 12 hours the following evening, and every evening thereafter until successful copulation occurred or the study had ended. As before, no food or water was provided during mating trials.

Upon review of the video recordings of subsequent mating trials, the following measures of female remating propensity were recorded relative to the start of the trial:

1) time at which females first mounted males and total number of mounts;

2) time at which successful copulation occurred (indicated by transfer of the spermatophore).

Analysis of variance was used to compare number of female mounts across treatments. Nonparametric failure-time analysis was used in comparisons of time to remating. This analysis provides a means of accommodating censored data, such as observations in which an event may not have occurred before the end of the study (Fox 1993). Data such as these are often omitted from any subsequent analyses, which can lead to biased comparisons (Fox 1993).

Hemolymph was extracted from adult males by severing one of the hind wings and using a microhematocrit capillary tube to draw hemolymph from the wound. Extraction of hemolymph from adult females was performed by making a small incision in the cuticle of the pronotum and drawing hemolymph from the wound with a micro-hematocrit tube. Wounds caused by these procedures normally heal very rapidly and the insects were observed to recover fully from their operations. None of the males used to obtain hemolymph were used in mating trials.

\section{RESULTS}

The results of our study show no significant differences in female sexual receptivity across treatments. Females that ingested male hemolymph had a slightly higher latency to remating on average, relative to females who received Ringer's solution and female hemolymph (Fig. 1). However, these differences were not statistically significant (Failuretime analysis: $\chi^{2}=2.68, \mathrm{P}=0.262$ ). Mean number of female mounts for each treatment did not differ significantly across treatments (ANOVA: $F_{2,21}=0.86$; $\mathrm{P}=0.481$; Fig. 2).

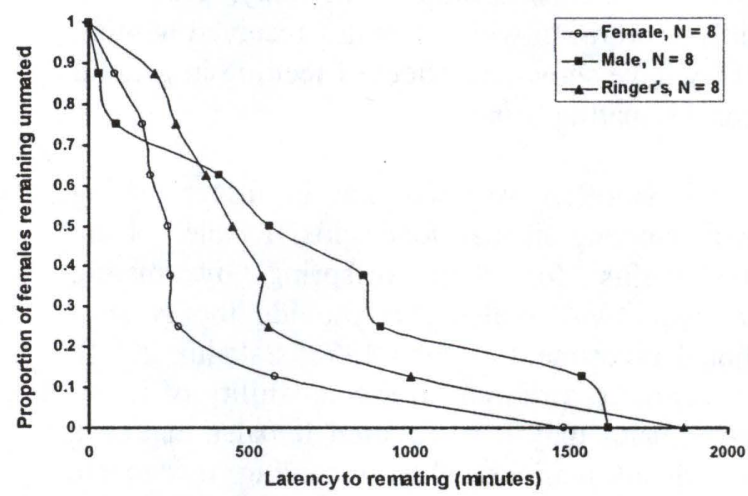

Figure 1: Survival distribution of latency to remating for females in each treatment. There was no significant difference in latency to remating across treatments.

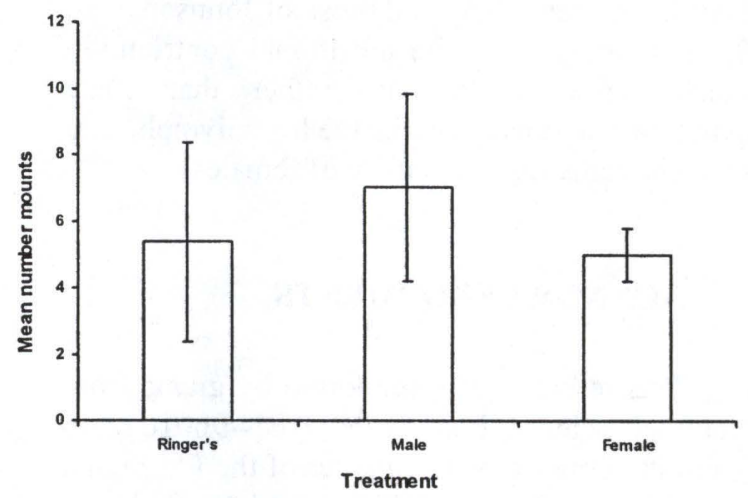

Figure 2: Mean number of female mounts $( \pm 1$ SE) for each treatment. There was no significant difference in mean number of female mounts across treatments.

\section{$\uparrow \quad$ DISCUSSION}

Female receptivity to remating was not affected by ingestion of male hemolymph prior to subsequent copulation. These results are inconsistent with the predictions of the male manipulation hypothesis and in apparent contradiction of the results of the Johnson et al. (1999) study. One explanation for this discrepancy is that any putative chemical or nutritional effects on female receptivity 
may be due to substances contained in the hind wing material rather than in the hemolymph of males. Our protocol did not account for this possibility as treatment substances consisted solely of hemolymph rather than wing material.

Females in the Johnston et al. (1999) study were exposed to only two treatments in initial matings: females received either a wing meal or no wing meal. Due to constraints of sample size and statistical power, we were hesitant to introduce any additional treatments in the present study. However, a negative control in which females received nothing at all may have shown an effect of feeding in general on female remating behavior.

It is often assumed that in insect mating systems involving nuptial food gifts, females obtain direct benefits for their offspring by mating preferentially with males that provide the greatest nutritional investment (Vahed 1998; Sakaluk 2000). In $C$. strepitans, variation in the capability of males to feed females may have favored females that seek out additional matings when receiving inadequate nourishment in previous matings. Such variation in male investment might be expected to arise as males continue to secure copulations and are gradually depleted of wing material and energy reserves. The results of the present study and those of Johnson et al. (1999) suggest that it is the nutritional contribution that males make at mating, rather than male manipulation via substances in the hemolymph, that influence the remating propensity of females.

\section{ACKNOWLEDGEMENTS}

This research was supported by grants from the National Science Foundation (IBN-9601042 to SKS) and the Beta Lambda Chapter of the Phi Sigma Biological Honor Society (CBW), and an R. Omar Rilett Research Scholarship from the Department of Biological Sciences at Illinois State University (CBW). Special thanks to Rachel Avery, Kevin Eckerle, Dr. John Hatle, and Dr. Hank Harlow for their help with the study.

\section{LITERATURE CITED}

Andersson, M. \& Iwasa, Y. 1996. Sexual selection. Trends in Ecology \& Evolution, 11: 53-58.
Dodson, G. N., Morris, G. K., \& Gwynne, D. T. 1983. Mating behavior in the primitive orthopteran genus Cyphoderris (Haglidae). In: Orthopteran mating systems: sexual competition in a diverse group of insects (D. T. Gwynne \& G. K. Morris, eds.), pp. 305318. Boulder, Colorado: Westview Press.

Eberhard. W. G. 1996. Female control: sexual selection by cryptic female choice. Princeton, New Jersey: Princeton University Press.

Eggert, A-K, Sakaluk, S. K. 1994. Sexual cannibalism and its relation to male mating success in sagebrush crickets, Cyphoderris strepitans (Haglidae: Orthoptera). Animal Behaviour, 47: 1171-1177.

Fox, G. A. 1993. Failure-time analysis: emergence, flowering, survivorship and other waiting times. In: Design and analysis of ecological experiments (S. M. Scheiner \& J. Gurevitch), pp. 253-289. New York: Chapman \& Hall.

Johnson, J. C., Ivy, T. M. \& Sakaluk, S. K. 1999. Female remating propensity contingent on sexual cannibalism in sagebrush crickets, Cyphoderris strepitans: a mechanism of cryptic female choice. Behavioral Ecology, 10: 227-233.

Sakaluk, S. K. 1984. Male crickets feed females to ensure complete sperm transfer. Science, 223: 609-610.

Sakaluk, S. K. 1991. Post-copulatory mate guarding in decorated crickets. Animal Behavior, 41: 207-216.

Sakaluk, S. K. 2000. Sensory exploitation as an evolutionary origin to nuptial food gifts in insects. Proceedings of the Royal Society of London, B, 267: 339-343.

Sakaluk, S. K. Bangert, P. J., Eggert, A-K, Gack, C., \& Swanson, L. V. 1995. The gin trap as a device facilitating coercive mating in sagebrush crickets. Proceedings of the Royal Society of London, B, 261: 65-71. 
Sakaluk, S. K. and Eggert, A.-K. 1996. Female control of sperm transfer and intraspecific variation in sperm precedence: antecedents to the evolution of a courtship food gift. Evolution, 50: 694-703.

Snedden, W. A. \& Sakaluk, S. K. 1992. Acoustical signaling and its relation to male mating success in sagebrush crickets. Animal Behaviour, 44: 633-639.
Thornhill, R., \& Alcock, J. 1983. The evolution of insect mating systems. Cambridge, MA: Harvard University Press.

Vahed, K. 1998. The function of nuptial feeding in insects: a review of empirical studies. Biological Reviews, 73: 43-78. 